\title{
ORIGINAL
}

\section{Valores normativos para evaluar la actitud hacia la obesidad en jóvenes universitarios}

\author{
Rossana Gomez-Campos ${ }^{a, b, c}$, Pablo Vasquez-Cerda ${ }^{d}$, Cristian Luarte-Rocha ${ }^{e}$, Vladimiro \\ Ibañez-Quispe ${ }^{f}$, Marco Cossio-Bolaños ${ }^{g, h, *}$
}

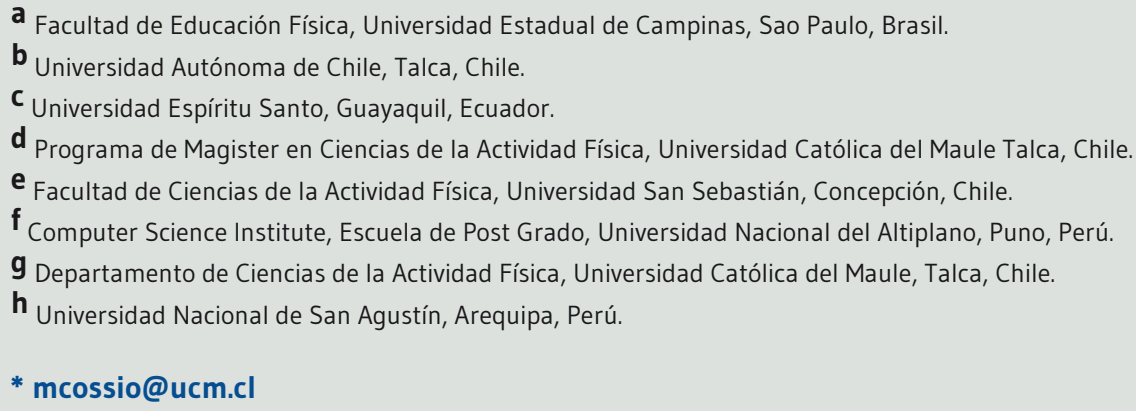

Recibido el 8 de noviembre de 2015; aceptado el 15 de enero de 2016.

Valores normativos para evaluar la actitud hacia la obesidad en jóvenes universitarios

\section{PALABRAS CLAVE}

Actitud;

Obesidad;

Estudiantes;

Universidades;

Estándar de referencia.

\section{RESUMEN}

Objetivos: Verificar la capacidad de reproducibilidad de una escala que valora la actitud a la obesidad y desarrollar estándares de percentil para jóvenes universitarios de la región del Maule (Chile).

Material y Métodos: Se efectuó una encuesta en 918 jóvenes universitarios (535 hombres y 383 mujeres) de la región del Maule (Chile). Se evaluó el peso y la estatura. Se calculó el Índice de Masa Corporal (IMC) y se aplicó una escala de actitud a la obesidad. Se utilizó el método LMS para desarrollar las curvas de percentil.

Resultados: La escala de actitud a la obesidad (EAO) mostró valores aceptables de Alfa de Cronbach (hombres 0,70) y mujeres 0,73). Las referencias de percentil fueron distribuidas en P15, P50 y P85 que permiten valorar por indicador (Preferencia/Rechazo, Preocupación/Desinterés y Voluntad/Apatía), rango de edad y sexo.

Conclusiones: La escala de actitud a la obesidad es un instrumento confiable y las referencias de percentiles propuestas son una alternativa para diagnosticar, controlar y monitorizar la actitud a la obesidad de jóvenes universitarios. 
Standard values to assess attitudes toward obesity in university students

\section{KEYWORDS}

Attitude;

Obesity;

Students:

Universities;

Reference

Standards.

\section{ABSTRACT}

Objective: Verifying the ability of reproducibility of a scale that assesses the attitude to obesity and develops percentile standard for university students from the region of Maule (Chile).

Material and Methods: A survey was conducted on 918 university students (535 men and 383 women) from the region of Maule (Chile). Weight and height were evaluated. Body mass index (BMI) was calculated and attitude scale was applied to obesity. The LMS method was used to develop percentile curves.

Results: The scale of attitude to obesity (EAO) showed acceptable values of Cronbach's alpha (0.70 men) and women 0.73). The percentile references were dealers in P15, P50 and P85 by allowing value indicator (Preference/Rejection, Concern/Selflessness and Will/Apathy), age range and gender.

Conclusions: The EAO is a reliable reference percentile and proposals are an alternative to diagnose, control and monitor obesity attitude of university students.

\section{CITA}

Gomez-Campos R, Vasquez-Cerda P, Luarte-Rocha C, Ibañez-Quispe V, Cossio-Bolaños M. Valores normativos para evaluar la actitud hacia la obesidad en jóvenes universitarios. Rev Esp Nutr Hum Diet. 2016 ; 20 (2): 104 - 110. doi: 10.14306/renhyd.20.2.193

\section{INTRODUCCIÓN}

La obesidad es una epidemia mundial grave y es una amenaza importante para la salud de los seres humanos ${ }^{1}$. Actualmente representa un problema multifactorial y puede ser debido a una combinación de factores: genéticos, del comportamiento, del medio ambiente, de la tecnología, de las oportunidades para la actividad física, de la accesibilidad, disponibilidad y economía?

Las estadísticas de sobrepeso y obesidad en los adultos a nivel mundial son alarmantes y las tendencias cada día van en alza. Por ejemplo, la Organización Mundial de la Salud proyectó para el 2015 aproximadamente 2,3 millones de adultos con sobrepeso y más de 700 millones con obesidad $^{3}$.

Al parecer estas tendencias tienen que ver mucho con la globalización, puesto que por un lado, ha mejorado sustancialmente la calidad de vida y la seguridad alimentaria, observándose reducción del nivel de pobreza en muchos países del mundo, y por otro lado, ha llevado a un aumento en el consumo de bebidas y comidas azucaradas con bajo contenido nutricional y un alto contenido de energía 4 , a esto se suman los reducidos niveles de actividad física, que en conjunto impulsan la epidemia mundial de la obesidad en todas las etapas de la vida.

En ese contexto, desde el punto de vista sociocultural, se debe tomar atención en los correlatos psicosociales relacionados con la estigmatización a la obesidad entre los adolescentes ${ }^{5}$ y jóvenes universitarios, puesto que en la vida cotidiana los jóvenes podrían desarrollar estereotipos negativos hacia la obesidad, expresando actitudes negativas hacia los estilos de vida saludables y consecuentemente descuidando su estado de salud. Por lo tanto, estas personas serían frecuentemente el blanco de la discriminación y los prejuicios en una variedad de entornos sociales y culturales, incluyendo áreas como el empleo, la educación y las relaciones interpersonales ${ }^{6}$, así como centros escolares y de educación superior.

Desde esa perspectiva, para ayudar a prevenir los drásticos incrementos de actitudes negativas hacia la obesidad entre los jóvenes universitarios, es importante contar con un instrumento válido y confiable que valore las actitudes hacia la obesidad; además es necesario disponer de estándares referenciales que permitan diagnosticar, controlar y monitorizar tales actitudes. 
En ese sentido, el presente estudio buscó, por un lado, verificar la capacidad de reproducibilidad de una escala que valora la actitud a la obesidad y, por otro lado, desarrollar estándares de percentil en jóvenes universitarios de la región del Maule (Chile). Esta información podría ayudar a utilizar diversas teorías y modelos educativos para diseñar y realizar intervenciones orientadas a lograr cambios de conducta que contribuyan a prevenir y controlar la obesidad y otras enfermedades crónicas?.

\section{METODOLOGÍA}

\section{Tipo de estudio y muestra}

Se efectuó una encuesta en 918 jóvenes universitarios (535 hombres y 383 mujeres) de la región del Maule (Chile). La selección de la muestra fue de tipo no probabilístico (cuotas). Los jóvenes seleccionados pertenecen a tres universidades de la ciudad de Talca y comprenden un rango de edad de 18-27 años. Las carreras profesionales que se consideraron en el estudio fueron: ciencias de la educación ( $n=367 ; 40 \%)$, ciencias de la salud ( $n=321 ; 35 \%)$ e ingenierías $(n=230 ; 25 \%)$.

Se incluyeron en el estudio a jóvenes que aceptaron voluntariamente la participación, a los que estaban dentro del rango edad establecida y a los que se encontraban estudiando entre el primer y cuarto año. Se excluyeron a los que no completaron el llenado del cuestionario y a los alumnos que se encontraban en el quinto año de estudio. La investigación tuvo la aprobación del comité de ética de la Universidad Autónoma (Talca, Chile).

\section{Procedimientos}

Para valorar la actitud hacia la obesidad se utilizó la técnica de la encuesta, cuyo instrumento fue una escala tipo Likert validada por Gómez et al. ${ }^{5}$ para adolescentes de la región del Maule (Chile). Este instrumento cuenta con tres indicadores (Preferencia/Rechazo, Preocupación/Desinterés y Voluntad/Apatía) los que se miden actitudes a través de 14 preguntas. La confiabilidad se verificó por medio de consistencia interna (Alfa de Cronbach).

Se midieron las variables antropométricas de peso y estatura y, por otro lado, la Escala de Actitud a la Obesidad (EAO). Ambos procedimientos de recolección de datos se efectuaron en un laboratorio de la Universidad Autónoma de Chile (Talca) entre las 9:00 y 13:00 horas y entre las 15:00 y 19:00 horas, de lunes a viernes durante los meses de junio y julio de 2015. La aplicación del instrumento se efectuó por grupos, durando aproximadamente entre 10-15 minutos. Las variables antropométricas se evaluaron siguiendo las recomendaciones de la Sociedad Internacional para los avances en Cinentropometría ${ }^{8}$. Se utilizó una báscula Tanita para el peso $(\mathrm{kg})$ con precisión de hasta $100 \mathrm{~g}$ y para la estatura un estadiómetro metálico de marca Seca con una precisión de $1 \mathrm{~mm}$ y una escala de 2,50m. Las características antropométricas se observan en la Tabla 1.

Todo el procedimiento estuvo a cargo de 4 evaluadores previamente entrenados y capacitados para medir variables antropométricas y técnicas de encuesta.

Se calculó el índice de masa corporal $\left(\mathrm{IMC}-\mathrm{kg} / \mathrm{m}^{2}\right)$ para relacionar el peso con la estatura y se utilizó la fórmula propuesta por Quetelet, donde: [IMC=Peso $\left.(\mathrm{kg}) / \operatorname{Estatura}(\mathrm{m})^{2}\right]$. La EAO puede ser interpretada según los percentiles como: baja actitud <P15; moderada actitud P15-P85; y alta actitud $>$ P85.

\section{Estadística}

La normalidad de la distribución de los datos se verificó por medio del test de Kolmogorov-Smirnov. Los resultados se analizaron por medio de estadística descriptiva de promedio, desviación estándar, rango, frecuencias, porcentajes y distribución de percentiles (P15, P50 y P85). Las diferencias entre ambos sexos se determinó por medio del test " $T$ " para muestras independientes ( $p<0,001)$. Se calculó el Alfa de Cronbach y los percentiles fueron determinados por medio del método $\mathrm{LMS}^{9}$. Esta técnica supone la existencia de tres curvas suavizadas: $L(t), M(t)$ y $S(t)$ (potencias de Box-Cox, medianas y coeficientes de variación), Los cálculos se realizaron en el programa LMS Chart Maker Light, versión 2.54. Todos los procedimientos de cálculo fueron realizados en planillas de Excel y en SPSS 18.0.

\section{RESULTADOS}

Las variables que caracterizan a la muestra del estudio se observan en la Tabla 1. Los hombres mostraron ser más altos y presentan mayor peso corporal en relación a las mujeres $(p<0,001)$. En el IMC, no hubo diferencias entre ambos sexos, excepto en el primer rango de edad (18-19 años), donde los hombres presentan menor IMC. En cuanto a los valores obtenidos en la EAO no hubo diferencias en ambos sexos y en todos los rangos de edad ( $p>0,001)$.

La Tabla 2 muestra los valores de consistencia interna por ítem y por sexo. En todos los casos el Alfa de Cronbach evidenció valores aceptables de confiabilidad. Los valores entre los ítems oscilan entre 0,61 y 0,76. En general, el instrumento mostró ser confiable $(\alpha=0,72)$. 
Tabla 1. Valores descriptivos de las variables antropométricas y de la Escala de Actitud hacia la Obesidad (EAO) de la muestra estudiada.

\begin{tabular}{|c|c|c|c|c|c|c|c|c|c|}
\hline \multirow{2}{*}{ Edades } & \multirow{2}{*}{$\mathrm{n}$} & \multicolumn{2}{|c|}{ Peso (kg) } & \multicolumn{2}{|c|}{ Estatura (cm) } & \multicolumn{2}{|c|}{ IMC (kg/m²) } & \multicolumn{2}{|c|}{ EAO } \\
\hline & & $\mathrm{X}$ & DE & $\mathrm{X}$ & DE & $\mathrm{X}$ & DE & $\mathrm{x}$ & DE \\
\hline \multicolumn{10}{|l|}{ Hombres } \\
\hline $18-19$ & 259 & 70,8 & $11,2^{*}$ & 172,1 & $6,8^{*}$ & 23,9 & $3,4^{*}$ & 26,3 & 3,5 \\
\hline $20-21$ & 92 & 72,8 & $10,1^{*}$ & 171,8 & $6,3^{*}$ & 24,6 & 2,4 & 25,9 & 3,4 \\
\hline $22-23$ & 72 & 77,1 & $12,3^{*}$ & 174,2 & $5,8^{*}$ & 25,3 & 3,0 & 25,9 & 3,7 \\
\hline $24-25$ & 62 & 77,6 & $14,9^{*}$ & 171,4 & $6,1^{*}$ & 26,3 & 3,9 & 25,8 & 3,4 \\
\hline $26-27$ & 50 & 75,7 & $6,9^{*}$ & 167,6 & $6,5^{*}$ & 27,0 & 2,3 & 24,8 & 4,4 \\
\hline Total & 535 & 73,3 & $12,1^{*}$ & 172,0 & $6,6^{*}$ & 24,7 & 3,5 & 26,0 & 3,5 \\
\hline \multicolumn{10}{|l|}{ Mujeres } \\
\hline $18-19$ & 131 & 64,2 & 12,6 & 159,0 & $6,1^{*}$ & 25,3 & 4,3 & 25,8 & 4,2 \\
\hline $20-21$ & 96 & 61,4 & 11,3 & 160,2 & $5,7^{*}$ & 24,0 & 4,4 & 25,6 & 4,7 \\
\hline $22-23$ & 65 & 65,2 & 15,8 & 156,8 & $6,2^{*}$ & 24,8 & 2,6 & 25,3 & 3,2 \\
\hline $24-25$ & 49 & 66,5 & 10,2 & 163,1 & $5,8^{*}$ & 25,0 & 4,0 & 26,3 & 3,8 \\
\hline $26-27$ & 42 & 68,2 & 13,6 & 160,0 & $7,1^{*}$ & 26,8 & 6,0 & 25,4 & 3,9 \\
\hline Total & 383 & 63,9 & 12,6 & 159,5 & $10,7^{*}$ & 25,4 & 10,2 & 25,7 & 4,1 \\
\hline
\end{tabular}

X: promedio; DE: Desviación estándar; IMC: Índice de Masa Corporal; Escala de Actitud hacia la Obesidad (EAO): expresado en puntos; *: diferencia significativa.

La propuesta de percentiles (P15, P50 y P85) para valorar la EAO se puede observar en la Tabla 3. Su valoración se puede efectuar por indicador, por rango de edad y sexo. Nótese que los valores de la mediana del indicador Preferencia/Rechazo son similares en ambos sexos. En el indicador Preocupación/Desinterés, los hombres presentan valores relativamente superiores en relación a las mujeres, sin embargo, en el indicador Voluntad/Apatía, las mujeres presentan ligera superioridad en comparación con los hombres. En general, los valores de la EAO son relativamente similares en ambos sexos y rangos de edad.

Los cálculos para estudios similares pueden ser efectuados en el siguiente link: http://reidebihu.net/cuesobeuni.php

\section{DISCUSIÓN}

En relación a la confiabilidad, los resultados del estudio evidenciaron moderada capacidad de reproducibilidad de la EAO. Los valores obtenidos de Alfa de Cronbach para ambos sexos oscilan entre $(0,70$ a 0,73$)$. Estos valores son consistentes con estudios que presentan similares propósitos, en el que verificaron la capacidad de reproducibilidad de escalas y/o cuestionarios asociadas a actitudes hacia la obesidad en adolescentes ${ }^{5,10}$, inclusive los a son similares con otros estudios que valoran la actividad física, conductas y hábitos alimentarios de adultos ${ }^{11-13}$.

De hecho, el Alfa de Cronbach es uno de los procedimientos más utilizados y preferidos por los estudios que utilizan técnicas de encuesta ${ }^{14}$, debido a su fácil aplicación y procesamiento. Además el uso de este estadígrafo desde su propuesta en 1951 ha crecido ostensiblemente en los últimos años. Prueba de ello es que se cita en las investigaciones un promedio aproximado de 960 veces por año ${ }^{15}$.

De acuerdo a los resultados observados, todos los elementos (ítems) contribuyen positivamente a la fiabilidad de la escala, a pesar de que se eliminaron preguntas $(\alpha=0,60$ $0,69)$ con el objetivo de elevar el $\alpha$, sin embargo, los resultados fueron similares. En ese contexto, se optó por mantener la estructura íntegra de la EAO (14 preguntas) basándose en las sugerencias de Sarkova et al ${ }^{16}$, en el que consideran que 
Tabla 2. Valores de confiabilidad por Alfa de Cronbach de la escala que valora la actitud hacia la obesidad.

\begin{tabular}{|c|c|c|c|c|}
\hline No: & Ítems & Hombres & Mujeres & Ambos \\
\hline \multicolumn{5}{|c|}{ A) Preferencia/Rechazo } \\
\hline 1 & Me agrada salir a reuniones sociales con mis amigos. & 0,712 & 0,769 & 0,740 \\
\hline 2 & Cuando tengo tiempo libre, prefiero mirar televisión en casa. & 0,744 & 0,757 & 0,741 \\
\hline 3 & $\begin{array}{l}\text { Cuando estoy en casa no me gusta ayudar en los quehaceres del hogar } \\
\text { (limpiar, barrer, planchar, cortar pasto, etc.). }\end{array}$ & 0,662 & 0,742 & 0,700 \\
\hline 4 & Cuando estoy en el colegio me gusta sentarme para conversar con mis amigos. & 0,722 & 0,776 & 0,750 \\
\hline \multicolumn{5}{|c|}{ B) Preocupación/Desinterés } \\
\hline 5 & ¿Te sentirías preocupado en el caso de que subas de peso repentinamente? & 0,699 & 0,688 & 0,640 \\
\hline 6 & ¿Alguna vez te has sentido intranquilo por tu barriga? & 0,618 & 0,698 & 0,666 \\
\hline 7 & Me preocupa mucho el hecho de no poder mantener mi peso ideal. & 0,720 & 0,695 & 0,699 \\
\hline 8 & Actualmente, ¿consideras que estás subiendo de peso? & 0,748 & 0,728 & 0,738 \\
\hline 9 & A menudo trato de no ingerir alimentos que me engorden. & 0,680 & 0,688 & 0,690 \\
\hline 10 & Me preocupo mucho cuando ingiero alimentos ricos en grasas. & 0,618 & 0,698 & 0,666 \\
\hline \multicolumn{5}{|c|}{ C) Voluntad/Apatía } \\
\hline 11 & Considero que tengo mucha voluntad para hacer ejercicios físicos. & 0,735 & 0,755 & 0,745 \\
\hline 12 & En general, considero que tengo autocontrol cuando ingiero mis alimentos. & 0,732 & 0,753 & 0,750 \\
\hline 13 & Pienso que soy capaz de mantener un estilo de vida saludable. & 0,731 & 0,743 & 0,733 \\
\hline 14 & $\begin{array}{l}\text { Considero que los ejercicios físicos son importantes para mantener mi peso } \\
\text { corporal. }\end{array}$ & 0,754 & 0,764 & 0,766 \\
\hline \multicolumn{2}{|c|}{ Alfa de Cronbach } & 0,70 & 0,73 & 0,72 \\
\hline
\end{tabular}

los valores de la subescalas deben ser superiores a 0,60 y las escalas totales son aceptables cuando superan el 0,70.

Por lo tanto, los valores de a no necesariamente deben ser altos, se estima que un máximo de 0,90 es recomendable para verificar la confiabilidad de los cuestionarios ${ }^{17}$, aunque desde el punto de vista del control de la calidad de los instrumentos es obligatorio estimar la exactitud (confiabilidad). Esto no sólo puede ser efectuado por la técnica de consistencia interna, sino también por mitades partidas y test re-test.

Respecto al desarrollo de estándares de percentil para valorar la actitud hacia la obesidad en jóvenes universitarios de la región del Maule, los resultados muestran que los percentiles son una alternativa para clasificar las actitudes de los jóvenes. Este estudio utilizó el método $\mathrm{LMS}^{9}$ para proponer los puntos de corte (P15, P50 y P85), pues los extremos de los centiles pueden ser estimados de forma más eficaz, además cualquier centil puede ser calculado y cada observación puede ser convertida a su desviación estándar ${ }^{18}$.
Los valores referenciales de la EAO pueden ser utilizados por rango de edad, sexo e indicadores (Preferencia/Rechazo, Preocupación/Desinterés y Voluntad/Apatía), pues estudios recientes a nivel mundial muestran su preocupación por cambiar las actitudes y percepciones negativas de las personas con sobrepeso y obesidad $5,19,20$. Por lo tanto, las referencias aquí propuestas son una alternativa para efectuar el seguimiento y vigilancia de la actitud hacia la obesidad de jóvenes universitarios de la región del Maule.

En ese contexto, los valores asociados a los percentiles superiores al P85 deben ser preocupantes, dado que estas actitudes están orientadas al rechazo, desinterés y apatía en relación a los estilos de vida saludables. Estas actitudes podrían llevar a adoptar una serie estereotipos negativos, comúnmente atribuidos a individuos obesos o con problemas de sobrepeso ${ }^{19}$, inclusive se ha llegado al extremo de denigrar y condenar a estas personas por su condición ${ }^{21}$. 
Rev Esp Nutr Hum Diet. 2016; 20(2): 104 - 110

Valores normativos para evaluar la actitud hacia la obesidad en jóvenes universitarios

Tabla 3. Distribución de percentiles de la actitud hacia la obesidad por rango de edad, sexo e indicadores.

\begin{tabular}{|c|c|c|c|c|c|c|c|c|c|c|c|c|c|}
\hline \multirow{2}{*}{ Edades } & \multicolumn{6}{|c|}{ Hombres } & \multicolumn{7}{|c|}{ Mujeres } \\
\hline & $\mathrm{L}$ & M & s & P15 & P50 & P85 & $\mathrm{L}$ & M & $\mathrm{s}$ & P5 & P15 & P50 & P85 \\
\hline \multicolumn{14}{|c|}{ Preferencia/Rechazo } \\
\hline $18-19$ & 1,60 & 8,73 & 0,18 & 7,0 & 8,7 & 10,2 & 1,31 & 8,96 & 0,18 & 6,1 & 7,2 & 9,0 & 10,6 \\
\hline $20-21$ & 0,78 & 8,09 & 0,18 & 6,6 & 8,1 & 9,6 & 1,04 & 8,55 & 0,19 & 5,9 & 6,9 & 8,6 & 10,2 \\
\hline $22-23$ & 0,39 & 8,22 & 0,18 & 6,8 & 8,2 & 9,9 & 0,84 & 8,35 & 0,19 & 5,9 & 6,8 & 8,3 & 10,0 \\
\hline $24-25$ & 0,64 & 8,72 & 0,19 & 7,1 & 8,7 & 10,4 & 0,74 & 8,83 & 0,18 & 6,3 & 7,2 & 8,8 & 10,5 \\
\hline $26-27$ & 1,16 & 8,73 & 0,19 & 7,0 & 8,7 & 10,4 & 0,83 & 8,43 & 0,20 & 5,8 & 6,7 & 8,4 & 10,2 \\
\hline
\end{tabular}

\section{Preocupación/Desinterés}

\begin{tabular}{l|l|l|l|l|l|l|l|l|l|l|l|l:l|l|l|l}
\hline $18-19$ & 0,43 & 11,36 & 0,26 & 8,5 & 11,4 & 14,7 & $-0,05$ & 9,93 & 0,29 & 6,2 & 7,4 & 9,9 & 13,4 \\
$20-21$ & 0,39 & 11,72 & 0,25 & 8,9 & 11,7 & 15,0 & 0,07 & 10,26 & 0,29 & 6,4 & 7,6 & 10,3 & 13,8 \\
$22-23$ & 0,22 & 11,25 & 0,24 & 8,7 & 11,3 & 14,4 & 0,12 & 10,48 & 0,28 & 6,5 & 7,8 & 10,5 & 14,0 \\
$24-25$ & $-0,05$ & 10,17 & 0,26 & 7,8 & 10,2 & 13,3 & 0,07 & 10,09 & 0,29 & 6,2 & 7,5 & 10,1 & 13,6 \\
$26-27$ & $-0,30$ & 9,76 & 0,27 & 7,5 & 9,8 & 13,1 & $-0,04$ & 9,84 & 0,31 & 6,0 & 7,2 & 9,8 & 13,6
\end{tabular}

\section{Voluntad/Apatía}

\begin{tabular}{|c|c|c|c|c|c|c|c|c|c|c|c|c|c|}
\hline 18-19 & $-0,29$ & 5,77 & 0,27 & 4,4 & 5,8 & 7,7 & 0,24 & 6,32 & 0,24 & 4,2 & 4,9 & 6,3 & 8,1 \\
\hline $20-21$ & $-0,34$ & 5,52 & 0,26 & 4,3 & 5,5 & 7,3 & 0,19 & 6,22 & 0,25 & 4,0 & 4,8 & 6,2 & 8,0 \\
\hline $22-23$ & $-0,17$ & 5,84 & 0,25 & 4,5 & 5,8 & 7,6 & 0,15 & 6,08 & 0,25 & 3,9 & 4,6 & 6,1 & 7,9 \\
\hline $24-25$ & 0,13 & 6,18 & 0,24 & 4,8 & 6,2 & 7,9 & 0,08 & 6,52 & 0,26 & 4,2 & 4,9 & 6,5 & 8,5 \\
\hline $26-27$ & 0,47 & 5,93 & 0,24 & 4,6 & 5,9 & 7,5 & $-0,02$ & 6,64 & 0,27 & 4,3 & 5,0 & 6,6 & 8,8 \\
\hline \multicolumn{14}{|l|}{ EAO } \\
\hline $18-19$ & 0,48 & 26,16 & 0,13 & 22,7 & 26,2 & 29,9 & $-0,04$ & 25,43 & 0,17 & 19,3 & 21,4 & 25,4 & 30,3 \\
\hline $20-21$ & 0,62 & 25,81 & 0,13 & 22,3 & 25,8 & 29,5 & 0,10 & 25,24 & 0,16 & 19,3 & 21,3 & 25,2 & 29,8 \\
\hline $22-23$ & 0,69 & 25,86 & 0,14 & 22,3 & 25,9 & 29,6 & 0,34 & 25,28 & 0,15 & 19,5 & 21,5 & 25,3 & 29,4 \\
\hline $24-25$ & 0,53 & 25,54 & 0,14 & 21,9 & 25,5 & 29,4 & 0,53 & 26,00 & 0,14 & 20,3 & 22,4 & 26,0 & 29,9 \\
\hline $26-27$ & 0,18 & 24,78 & 0,14 & 21,3 & 24,8 & 28,7 & 0,84 & 25,58 & 0,13 & 20,1 & 22,1 & 25,6 & 29,2 \\
\hline
\end{tabular}

L: asimetría; M: mediana; S: coeficiente de variación; EAO: Escala de Actitud hacia la Obesidad.

En suma, tanto la EAO y las referencias propuestas deben servir a los jóvenes universitarios para adoptar actitudes relacionadas con las preferencias, con la preocupación y la fuerza de voluntad de actividades que conllevan a preservar un adecuado estado de salud, a pesar de que en la actualidad los jóvenes tienen que lidiar con los factores psicológicos, sociales, culturales y económicos ${ }^{22}$, que muy bien pueden influir en sus actitudes.
El estudio en general presenta algunas fortalezas, como el tamaño de la muestra utilizada y la capacidad de fiabilidad que evidenció el instrumento. Las referencias expresadas en percentiles permiten identificar a individuos con posibles actitudes hacia la obesidad, lo que evidentemente va en perjuicio de su estado de salud. Se sugiere para futuros estudios utilizar la EAO en otras muestras de jóvenes universitarios, cuyo objetivo principal sea comprobar la validez externa. 


\section{CONCLUSIONES}

La EAO es un instrumento confiable y las referencias de percentiles propuestas son una alternativa para diagnosticar, controlar y monitorizar la actitud hacia la obesidad de jóvenes universitarios. Los resultados sugieren su uso y aplicación de forma rutinaria en las universidades de la región.

\section{AGRADECIMIENTOS}

Los autores agradecen el financiamiento del proyecto a la Universidad Autónoma - Proyecto Interno N DIP115-16 y al Fondecyt Regular - N 1141295.

\section{$\longrightarrow$ \\ CONFLICTO DE INTERESES}

Los autores expresan que no hay conflictos de intereses al redactar el manuscrito.

\section{REFERENCIAS}

(1) Zhang Y, Liu J, Yao J, Ji G, Qian L, Wang J, et al. Obesity: pathophysiology and intervention. Nutrients. 2014; 6(11): 5153-83.

(2) Kopelman P, Jebb SA, Butland B. Executive summary: Foresight «Tackling Obesities: Future Choices» project. Obes Rev. 2007; 8 Suppl 1: vi-ix.

(3) Cho J, Juon H-S. Assessing overweight and obesity risk among Korean Americans in California using World Health Organization body mass index criteria for Asians. Prev Chronic Dis. 2006; 3(3): A79.

(4) Malik VS, Willett WC, Hu FB. Global obesity: trends, risk factors and policy implications. Nat Rev Endocrinol. 2013; 9(1): 13-27.

(5) Gómez R, de Arruda M, Sulla J, Alvear F, Urra C, González C, et al. Construcción de un instrumento para valorar la actitud a la obesidad en adolescentes. Rev Chil Nutr. 2015; 42(3): 277-83.

(6) Puhl R, Brownell KD. Bias, discrimination, and obesity. Obes Res. 2001; 9(12): 788-805.

(7) Olivares S, Bustos N, Moreno X, Lera L, Cortez S. Food and physical activity attitudes and practices in obese children and their mothers in Santiago, Chile. Rev Chil Nutr. 2006; 33(2): 170-9.
(8) Stewart A, Marfell-Jones M. International standards for anthropometric assessment. ISAK, editor. Lower Hutt, New Zealand: International Society for the Advancement of Kinanthropometry; 2011.

(9) Cole TJ, Bellizzi MC, Flegal KM, Dietz WH. Establishing a standard definition for child overweight and obesity worldwide: international survey. BM]. 2000; 320(7244): 1240-3.

(10) Gago J, Tornero I, Carmona J, Sierra Á. Actitudes hacia la obesidad de los estudiantes de Magisterio de la Universidad de Huelva. RETOS. 2012; (22): 38-42.

(11) Fernández Cabrera T, Medina Anzano S, Herrera Sánchez IM, Rueda Méndez S, Fernández Del Olmo A. Construcción y validación de una escala de autoeficacia para la actividad física. Rev Esp Salud Publica. 2011; 85(4): 405-17.

(12) Pino JL, Díaz C, López MÁ. Construcción y validación de un cuestionario para medir conductas y hábitos alimentarios en usuarios de la atención primaria de salud. Rev Chil Nutr. 2011; 38(1): 41-51.

(13) Gómez Campos R, de Arruda M, Camargo C, Cossio Bolaños MA. Confiabilidad de un cuestionario que valora la actividad física en adolescentes normopeso y con exceso de peso. Nutr Hosp. 2015; 31(5): 2205-11.

(14) Cossio-Bolaños M, Viveros-Flores A, Castillo-Retamal M, Vargas-Vitoria R, Gatica P, Gómez-Campos R. Patrones de actividad física en adolescentes en función del sexo, edad cronológica y biológica. Nutr clín diet hosp. 2015; 35(2): 41-7.

(15) Harzing AW. Publish or Perish [Internet]. 2007. Recuperado a partir de: http://www.harzing.com/pop.htm

(16) Sarkova M, Nagyova I, Katreniakova Z, Geckova AM, Orosova 0 , Middel B, et al. Psychometric evaluation of the general health questionnaire-12 and Rosenberg self-esteem scale in Hungarian and Slovak early adolescents. STUDIA PSYCHOLOGICA. 2006; 48(1): 69-79.

(17) Streiner DL. Starting at the beginning: an introduction to coefficient alpha and internal consistency. J Pers Assess. 2003; 80(1): 99-103.

(18) Kułaga Z, Litwin M, Tkaczyk M, Palczewska I, Zajączkowska M, Zwolińska D, et al. Polish 2010 growth references for schoolaged children and adolescents. Eur J Pediatr. 2011; 170(5): 599-609.

(19) Schwartz MB, Vartanian LR, Nosek BA, Brownell KD. The influence of one's own body weight on implicit and explicit anti-fat bias. Obesity (Silver Spring). 2006; 14(3): 440-7.

(20) Vartanian LR, Silverstein KM. Obesity as a status cue: perceived social status and the stereotypes of obese individuals. J Appl Soc Psychol. 2013; 43: E319-28.

(21) Rogge MM, Greenwald M, Golden A. Obesity, stigma, and civilized oppression. ANS Adv Nurs Sci. 2004; 27(4): 301-15.

(22) Cossio-Bolaños MA, de Arruda M, Moyano A, Gañán E, Pino LM, Lancho JL. Composición corporal de jóvenes universitarios en relación a la salud. Nutr clín diet hosp. 2011; 31(3): 15 